\title{
On the Role of Transformations Destroying Analyticity
}

\author{
Claus Kahlert* and Michael Klein \\ Institute for Physical and Theoretical Chemistry University of Tübingen \\ Z. Naturforsch. 43a, $1091-1093$ (1988); received September 24, 1988

\begin{abstract}
Nonconformal transformations, which leave the qualitative behavior of discrete dynamical sy-
\end{abstract} \\ stems invariant but destroy or create analyticity for the equations of motion are investigated. For \\ linear transformations, an explicit criterion is demonstrated, which allows to check whether a map \\ is conjugate to a complex holomorphic dynamics.
}

\section{On the Role of Transformations Destroying Analyticity}

Recently the question of fractal basin boundaries [1], specifically in real (non-conformal) 2-D discrete dynamical systems gained much interest [2-4]. To our opinion, the role of analyticity in iterated (complex) mappings, i.e., dynamical systems, is only very poorly understood at the moment. On the one hand, conformal structures yield a very powerful tool for investigating Julia sets [5] or the Mandelbrot set [6], on the other hand, the Cauchy-Riemann conditions (cf. e.g. [7]) are by no means necessary for obtaining structures which are indistinguishable from, say, those concerning the complex logistic map $z \rightarrow z^{2}+c$. An example of a two-dimensional real map [8], based on a physical interpretation, turned out to be related to the latter dynamics via the simple transformation $x=x^{\prime}, y=a \cdot y^{\prime}$ (where $x=\mathfrak{R} z$ and $y=\mathfrak{J} z$ ).

Although the mentioned transform causes just a stretching of one coordinate, it is sufficient to destroy the holomorphic character of the dynamics - without changing any quality of the dynamical behavior, including the structure of the Mandelbrot set. Thus, at a first stage, we would like to address the question: Is it possible to find out whether a two-dimensional real dynamical system behaves equivalently to a complex analytic one, i.e., can it be transformed to a system from the latter class?

Since we are only interested in the qualitative dynamical behavior of a system, any sufficiently smooth automorphism (say, on the Riemann sphere), has to be

* Current address: Electronics Research Laboratory, University of California, Berkeley, CA 94720, USA.

Reprint requests to Michael Klein, Institut für Physikalische und Theoretische Chemie, Universität Tübingen, Auf der Morgenstelle 8, D-7400 Tübingen. taken into consideration. As an example, the basins of attraction of a cycle of period 11 and of infinity for the complex logistic map are presented - with coordinates transformed via the Henon map. That is, the structures shown here are nothing but the well known results from the $z^{2}$-family - provided the current transformation is on hand. To our knowledge there exists no theorem which allows one to decide whether a dynamical system can be transformed to a complex analytic one or not, thus even the question of existence is open. However, we are not going to get deeper into the problem of general transformations but rather turn to the specific question of finding a criterion for linear transforms.

Any homogeneous linear automorphism, T, in two real dimensions (trivial shifts will be neglected) may be written as

$$
T=\alpha \cdot\left(\begin{array}{ll}
1 & 0 \\
a & b
\end{array}\right)\left(\begin{array}{rr}
\cos \varphi & -\sin \varphi \\
\sin \varphi & \cos \varphi
\end{array}\right)=\alpha \mathbf{S ~ C .}
$$

The constant factor $\alpha$ together with the second matrix, C, yield the general form of a conformal linear transform, and hence do not change the analyticity of a map. In contrast, the first transform, $\mathbf{S}$, is either a diagonal matrix with $b= \pm 1$, which leaves the holomorphic structure of the dynamics invariant, or it is a non-conformal transform, that might destroy analyticity or allows to regain it. Since any linear mapping possesses the shape of the first matrix, modulo a conformal transform, for our present purpose we can use $\mathbf{S}$ as the canonical representation of a linear automorphism.

Let the equation of motion be given by

$$
\begin{aligned}
& x_{n+1}^{\prime}=F^{\prime}\left(x_{n}^{\prime}, y_{n}^{\prime}\right), \\
& y_{n+1}^{\prime}=G^{\prime}\left(x_{n}^{\prime}, y_{n}^{\prime}\right) .
\end{aligned}
$$

0932-0784/88/1200-1091 \$01.30/0. - Please order a reprint rather than making your own copy. 

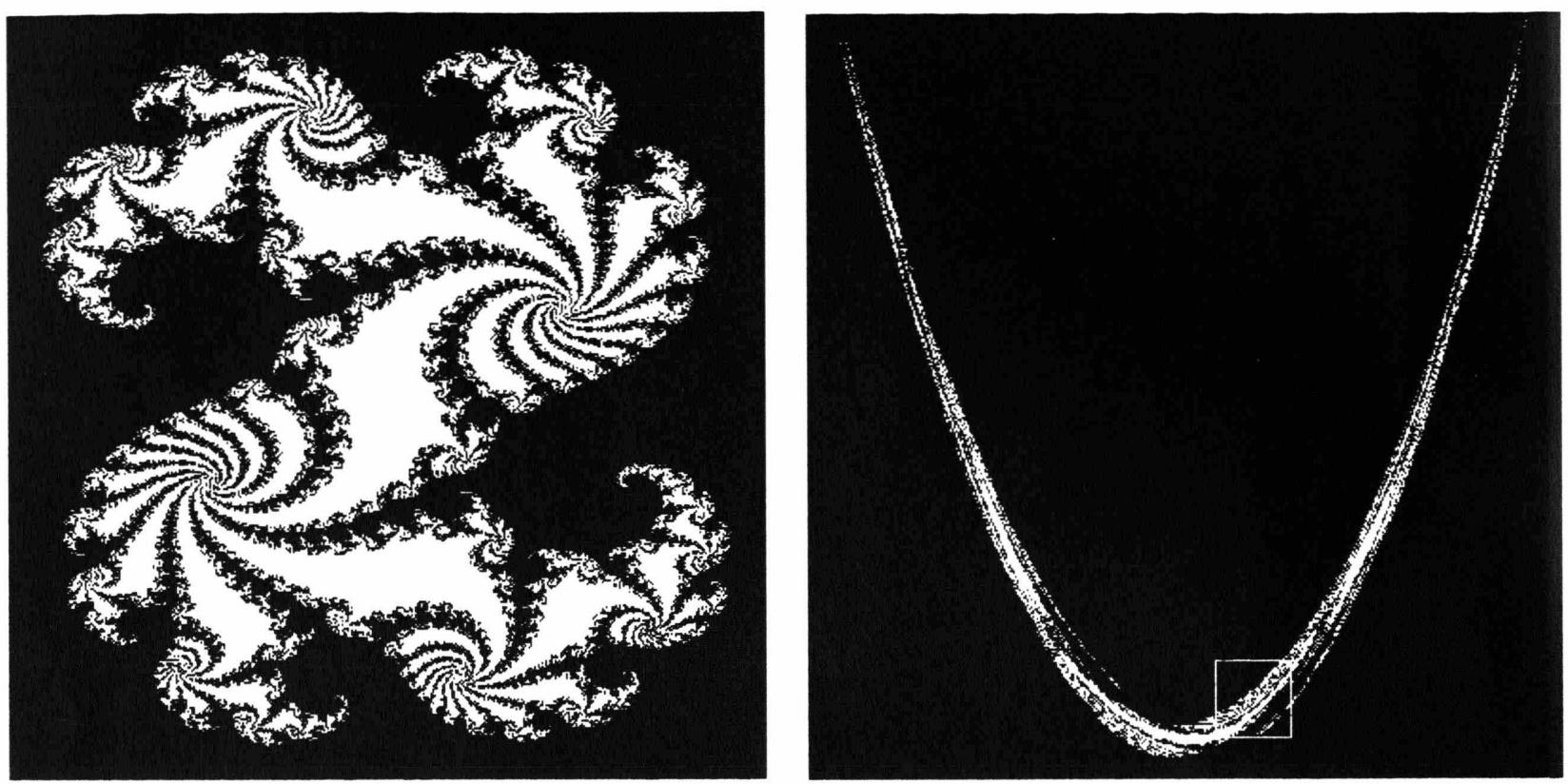

a)
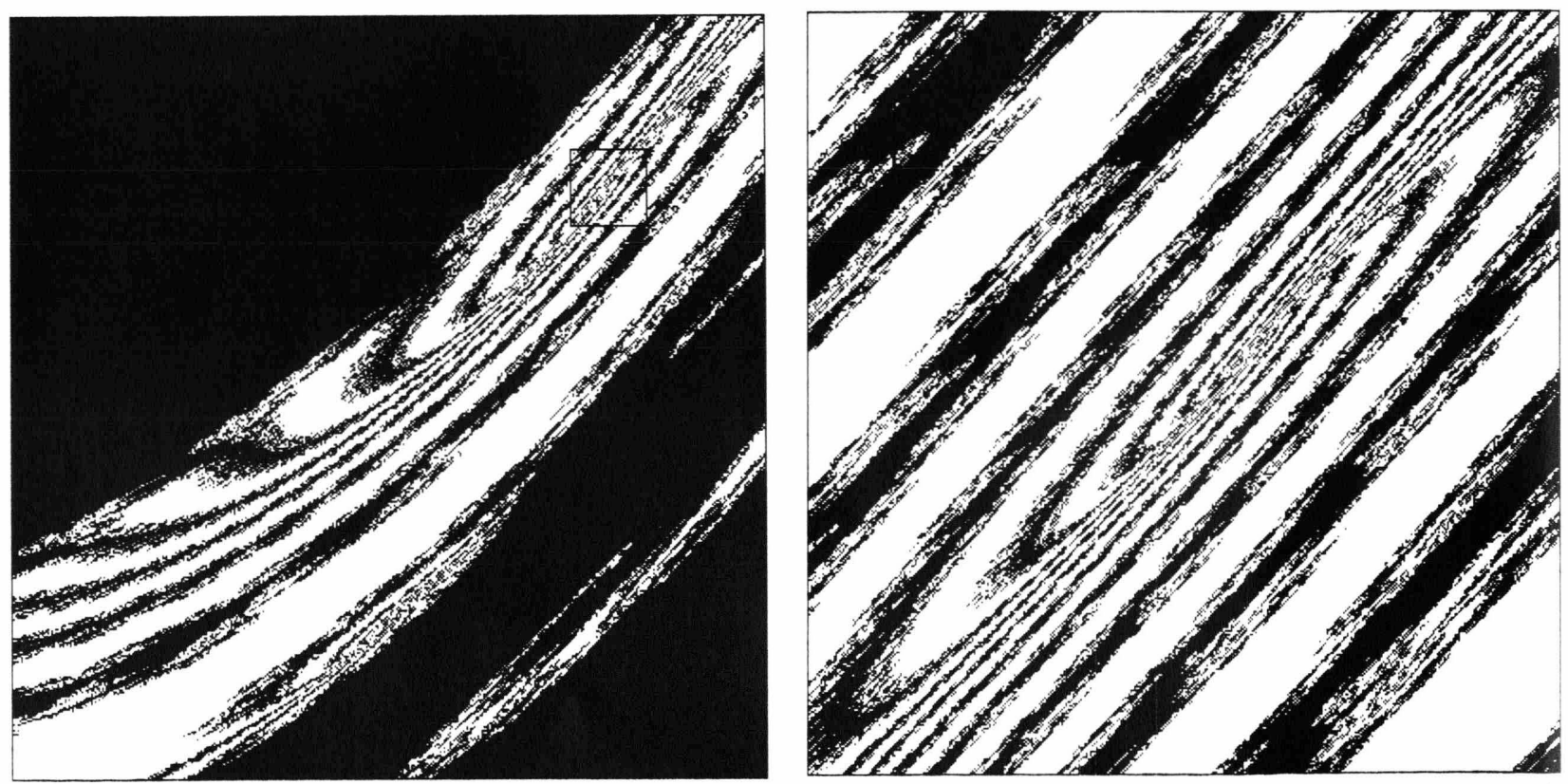

c)

Fig. 1. The basins of attraction of a cycle of period 11 (white) and of infinity (black) for the dynamics $x_{n+1}=x_{n}^{2}-y_{n}^{2}+c_{1}$, $y_{n+1}=2 x_{n} y_{n}+c_{2}$ (the complex logistic map) (part a) and for $x_{n+1}^{\prime}=2 x_{n}^{\prime} \cdot\left(1-p_{1} x_{n}^{\prime 2}+y_{n}^{\prime}\right)+p_{4}, y_{n+1}^{\prime}=\left(1-p_{1} x_{n}^{\prime 2}+y_{n}^{\prime}\right)^{2}$ $-p_{2}^{2} x_{n}^{\prime 2}+p_{3}-1+p_{1} \cdot\left(2 x_{n}^{\prime}\left(1-p_{1} x_{n}^{\prime 2}+y_{n}^{\prime}\right)+p_{4}\right)^{2}$ (parts b to d), being a nonlinear transform of the first one via the Henon map $\left(x=1-p_{1} x^{\prime}+y^{\prime}, y=p_{2} x^{\prime}\right)$. If only the second dynamics is available, it will be very hard to find out that it is equivalent to the first one. Parameters: $c_{1}=0.32, c_{2}=0.043 ; p_{1}=1.4, p_{2}=0.3, p_{3}=0.32, p_{4}=0.14 \overline{3}$. Axes (a) $x=-0.9 \ldots 0.9$, $y=-1.2 \ldots 1.2 ; \quad$ (b) $x^{\prime}=-4.0 \ldots 4.0, \quad y^{\prime}=-2.0 \ldots 18.0 ; \quad$ (c) $x^{\prime}=0.3 \ldots 1.1, \quad y^{\prime}=-0.9 \ldots 1.1 ; \quad$ (d) $x^{\prime}=0.896 \ldots 0.936$, $y^{\prime}=0.55 \ldots 0.65$. 
Via $\mathbf{S}$ it is transformed to

$$
\begin{aligned}
x_{n+1}= & F\left(x_{n}, y_{n}\right)=F^{\prime}\left(x_{n}, a x_{n}+b y_{n}\right) \\
y_{n+1}= & G\left(x_{n}, y_{n}\right)=-\frac{a}{b} F^{\prime}\left(x_{n}, a x_{n}+b y_{n}\right) \\
& +\frac{1}{b} G^{\prime}\left(x_{n}, a x_{n}+b y_{n}\right) .
\end{aligned}
$$

Now in order for the transformed dynamics $(F(x, y)$, $G(x, y))$ to satisfy the Cauchy-Riemann equations $\frac{\partial F}{\partial x}=\frac{\partial G}{\partial x}$ and $\frac{\partial F}{\partial y}=-\frac{\partial G}{\partial x}$, the conditions

$$
F_{x^{\prime}}^{\prime}+a F_{y^{\prime}}^{\prime}=-a F_{y^{\prime}}^{\prime}+G_{y^{\prime}}^{\prime}
$$

and

$$
b F_{y^{\prime}}^{\prime}=\frac{a}{b} F_{x^{\prime}}^{\prime}+\frac{a^{2}}{b} F_{y^{\prime}}^{\prime}-\frac{1}{b} G_{x^{\prime}}^{\prime}-\frac{a}{b} G_{y^{\prime}}^{\prime}
$$

(where $F_{x^{\prime}}^{\prime}=\partial F^{\prime} \partial \partial x^{\prime}$, etc.) have to be fulfilled. These equations can be solved easily, yielding

$$
a=\frac{G_{y^{\prime}}^{\prime}-F_{x^{\prime}}^{\prime}}{2 F_{y^{\prime}}^{\prime}}
$$

as well as

$$
b= \pm \sqrt{\frac{a F_{x^{\prime}}^{\prime}+a^{2} F_{y^{\prime}}^{\prime}-G_{x^{\prime}}^{\prime}-a G_{y^{\prime}}^{\prime}}{F_{y^{\prime}}^{\prime}} .}
$$

The two possible signs of $b$ reflect the fact that the matrix $\left(\begin{array}{rr}1 & 0 \\ 0 & -1\end{array}\right)$ is not a conformal map, but, together with its inverse, leaves the holomorphic structure unaffected. Equation (5) provides several conditions on the original dynamics in order to make $\mathbf{S}$ an automorphism: First the two expressions on the righthand sides have to be real constants, i.e., they must be

[1] S. W. McDonald, C. Grebogi, E. Ott, and J. A. Yorke, Physica 17 D, 125 (1985).

[2] O. E. Rössler, C. Kahlert, J. Parisi, J. Peinke, and B. Röhricht, Z. Naturforsch. 41 a, 819 (1986).

[3] O. E. Rössler, J. L. Hudson, J. A. Yorke, Z. Naturforsch. 41 a, 979 (1986).

[4] C. Kahlert and O. E. Rössler, Z. Naturforsch. 42a, 324 (1987).

[5] G. Julia, J. Math. Pure et Appl. 8, 47 (1918).

[6] B. B. Mandelbrot, The Fractal Geometry of Nature, Freeman, San Francisco 1982 independent both of $x^{\prime}$ and of $y^{\prime}$. For this purpose $F_{y^{\prime}}^{\prime}$ may not vanish. Moreover $b$ too has to be nonzero, otherwise $\mathbf{S}$ would be a singular matrix.

For the special cases of already holomorphic dynamics, the matrix $\mathbf{S}$ turns out to be of the shape $\left(\begin{array}{rr}1 & 0 \\ 0 & \pm 1\end{array}\right)$, i.e., it is either the identity mapping or it switches the orientation of the coordinate frame. And for those dynamics where coordinates are just stretched without changing the relative direction (like in the example cited above), we find $a=0$ and $b= \pm \sqrt{-G_{x^{\prime}}^{\prime} / F_{y^{\prime}}^{\prime}}$.

Now that we have solved one problem, two others arose. Analyticity of the map is definitly not a property of any direct relevance for the dynamical behavior of a system. However, it is still an open question whether bi-fractal [9] basin boundaries appear only in dynamics being conjugate to a complex analytic one. This is tightly connected to the second problem of finding a possible transformation which restores the Cauchy-Riemann property. Although both questions are still open, it seems no longer justified to talk about the dynamical structures which arise in the complex analytic case, as being limited to "very special cases of two-dimensional maps" [1]. On the contrary we see the chance that the dynamics of a huge class of dynamical systems can be treated by the extremely powerful tool of the theory of holomorphic complex functions.

\section{Acknowledgement}

We both thank Otto Rössler and Jack Hudson for discussion. C. K. gratefully acknowledges support from the Alexander von Humboldt-Foundation.

[7] E. C. Titchmarsh, Theory of Functions, University Press, Oxford 1939.

[8] M. Klein, Z. Naturforsch. 43a, (1988), in press.

[9] B. Röhricht, W. Metzler, J. Parisi, J. Peinke, W. Beau, and O. E. Rössler, The Classes of Fractals, The Physics of Structure Formation, W. Güttinger and G. Dangelmayr, Editors, Springer Series in Synergetics 37, Springer-Verlag, Berlin 1987, pp. 275-281. 